\title{
Expression and Purification of the Recombinant Pseudomonas Exotoxin A Conjugated to Herceptin and Its Anti-Proliferation Effects on Sk-Br-3
}

\author{
Maryam Hemmati ${ }^{1}$, Parastoo Tarighi ${ }^{1}$, Sahel Amoozadeh ${ }^{1}$, \\ Mohammad Morad Farajollahi ${ }^{1, *}$ \\ ${ }^{1}$ Department of medical biotechnology, Faculty of allied medicine, Iran University of \\ medical sciences, Tehran, Iran
}

* Corresponding author: Mohammad Morad Farajollahi, Department of medical

DOI: $10.21859 /$ mci-supp-33 biotechnology, Faculty of allied medicine, Iran University of medical sciences, Tehran,

\section{Keywords:}

Pseudomonas

Exotoxin A

Sk-Br-3
Iran.E-mail:mahdiy@yahoo.com

\begin{abstract}
Introduction Breast cancer is one of the most common cancers among women. There are many different therapies for treating breast cancer that targeted therapy is one of them. Herceptin is a monoclonal antibody against Her2-neu receptor that is over expressed in some breast cancer cell lines that is used in targeted therapy. This drug is expensive, has lots of side effects, and has low efficiency. Immunotoxins are another kind of targeted therapy that results in the binding of a toxin to one of the immune systems components such as antibodies. In this study we introduce an immunotoxin consisting of a truncated pseudomonas exotoxin A conjugated to Herceptin antibody in order to improve the efficiency of the Herceptin drug.

Materials and Methods: We eliminated the binding domain sequence of Pseudomonas exotoxin A and inserted into pET28a expression vector then transformed in an E.coli strain BL21 (DE3).The truncated toxin was expressed then was isolated through nickel-sepharose chromatography method. Herceptin and the toxin were conjugated through aldehydes bonding. The effects of this immunotoxin, herceptin and toxin were separately tested on SK-BR3 Her2 positive cell lines then MTT assay was performed for cell viability.

Results: The cytotoxicity of Herceptin-truncated exotoxin A was significantly higher in comparison to toxin and antibody alone on SK-BR3 cell line.

Conclusions: Truncated toxin-Herceptin bio conjugation can be a potential candidate with increased efficiency for treating breast cancer patients with over expression of the HER2-neu receptor.
\end{abstract}

